\title{
An existence and uniqueness theorem for the Navier-Stokes equations in dimension four
}

\section{Vincenzo Coscia}

To cite this article: Vincenzo Coscia (2016): An existence and uniqueness theorem for the Navier-Stokes equations in dimension four, Applicable Analysis, DOI: 10.1080/00036811.2016.1263837

To link to this article: http://dx.doi.org/10.1080/00036811.2016.1263837

曲 Published online: 02 Dec 2016.

Submit your article to this journal $[\pi$

山 Article views: 17

Q View related articles $\asymp$

View Crossmark data 


\section{An existence and uniqueness theorem for the Navier-Stokes equations in dimension four}

\section{Vincenzo Coscia}

Dipartimento di Matematica e Informatica, Università di Ferrara, Ferrara, Italy

\section{ABSTRACT}

We prove that the steady state Navier-Stokes equations have a solution in an exterior Lipschitz domain of $\mathbb{R}^{4}$, vanishing at infinity, provided the boundary datum belongs to $L^{3}(\partial \Omega)$.

\section{ARTICLE HISTORY}

Received 22 October 2016

Accepted 18 November 2016

\section{COMMUNICATED BY}

G. Panasenko

\section{KEYWORDS}

Stationary Navier Stokes equations; $4 D$ exterior

Lipschitz domains;

boundary-value problem

\section{AMS SUBJECT}

CLASSIFICATIONS

76D05; 35Q30; 31B10; 76D03

\section{Introduction}

In this paper, we shall consider the steady-state boundary-value problem for the Navier-Stokes equations ${ }^{1}$

$$
\begin{aligned}
v \Delta \boldsymbol{u}-\boldsymbol{u} \cdot \nabla \boldsymbol{u}-\nabla p & =\mathbf{0} & & \text { in } \Omega, \\
\operatorname{div} \boldsymbol{u} & =0 & & \text { in } \Omega, \\
\boldsymbol{u} & =\boldsymbol{a} & & \text { on } \partial \Omega \\
\lim _{r \rightarrow+\infty} \boldsymbol{u}(x) & =\mathbf{0} & & \text { on } \partial \Omega
\end{aligned}
$$

in the four-dimensional exterior domain

$$
\Omega=\mathbb{R}^{4} \backslash \bigcup_{i=1}^{m} \bar{\Omega}_{i}
$$

where $\Omega_{i}$ are bounded Lipschitz domains with connected boundaries such that $\Omega_{i} \cup \Omega_{j}=\varnothing, i \neq j$. In (1), $\boldsymbol{u}$ and $p$ are the kinetic and pressure fields respectively, $v>0$ is the kinematical viscosity coefficient and $\boldsymbol{a}$ is an assigned field on $\partial \Omega$.

Strictly connected to (1) is its linearized version, the Stokes equations

$$
\begin{aligned}
v \Delta \boldsymbol{u}-\nabla p=\mathbf{0} & \text { in } \Omega, \\
\operatorname{div} \boldsymbol{u}=0 & \text { in } \Omega, \\
\boldsymbol{u}=\boldsymbol{a} & \text { on } \partial \Omega \\
\lim _{r \rightarrow+\infty} \boldsymbol{u}(x)=\mathbf{0} & \text { on } \partial \Omega .
\end{aligned}
$$


It is well known that if $\boldsymbol{a} \in L^{3}(\partial \Omega)$, then (2) has a solution ${ }^{2}\left(\boldsymbol{u}_{s}, p_{s}\right)$ analytical in $\Omega$, such that $\boldsymbol{u}_{s}=O\left(r^{-2}\right), p_{s}=O\left(r^{-3}\right)$ and which satisfies $(2)_{3}$ in the sense of the non tangential convergence, i.e, there is a finite cone $\Gamma$ such that

$$
\lim _{x\left(\in \Gamma_{\xi}\right) \rightarrow \xi} \boldsymbol{u}(x)=\boldsymbol{a}(\xi)
$$

for almost all $\xi \in \partial \Omega$, where $\Gamma_{\xi} \subset \Omega$ is a cone with vertex at $\xi$, congruent to $\Gamma$.

In Section 3, we prove the following

Theorem 1: If $\boldsymbol{a} \in L^{3}(\partial \Omega)$ and

$$
\mathcal{F}=\frac{1}{4 \omega} \sum_{i=1}^{m}\left|\int_{\partial \Omega_{i}} \boldsymbol{a} \cdot \boldsymbol{n}\right| \max _{\partial \Omega} \frac{1}{\left|x-x_{i}\right|^{2}}<v,
$$

where $\omega$ is the measure of the surface of the unit ball of $\mathbb{R}^{4}, x_{i}$ fixed point of $\Omega_{i}$, and $\boldsymbol{n}$ is the outward (with respect to $\Omega$ ) unit normal to $\partial \Omega$, then (1) has a solution $(\boldsymbol{u}, p) \in\left[L^{4}(\Omega) \cap C^{\infty}(\Omega)\right] \times C^{\infty}(\Omega)$. Moreover, $\boldsymbol{u}$ is unique in $L^{4}(\Omega)$, provided $3\left\|\boldsymbol{u}_{s}\right\|_{L^{4}(\Omega)}<4 v$ and

$$
\left\|\boldsymbol{u}_{s}\right\|_{L^{4}(\Omega)}+\frac{\left\|\boldsymbol{u}_{s}\right\|_{L^{4}(\Omega)}^{2}}{\frac{4}{3}\left(v-\frac{3}{4}\left\|\boldsymbol{u}_{s}\right\|_{L^{4}(\Omega)}\right)}<\frac{4 v}{3},
$$

where $\boldsymbol{u}_{s}$ is the solution to (2) in $\Omega$ with boundary datum $\boldsymbol{a}$.

In the next section, we collect the main preliminary tools we shall need to get our results. For domains of class $C^{1,1}$ and boundary data in $W^{1 / 4,4}(\partial \Omega)$ problem (2) in bounded domains has been considered by several authors (see p.297 of [1] and the references therein).

\section{Preliminary results}

The fundamental solution to (2) writes

$$
\begin{aligned}
& \mathcal{U}_{i j}(x-y)=-\frac{1}{4 \omega \nu|x-y|^{2}}\left\{\delta_{i j}+\frac{2\left(x_{i}-y_{i}\right)\left(x_{j}-y_{j}\right)}{|x-y|^{2}}\right\}, \\
& \varpi_{i}(x-y)=\frac{x_{i}-y_{i}}{\omega|x-y|^{4}} .
\end{aligned}
$$

The Stokes simple layer potential with density $\psi \in L^{q}(\partial \Omega)$ is defined by

$$
\begin{gathered}
\boldsymbol{v}[\boldsymbol{\psi}](x)=\int_{\partial \Omega} \mathfrak{u}(x-\xi) \cdot \boldsymbol{\psi}(\xi) \mathrm{da} \xi \\
P[\boldsymbol{\psi}](x)=\int_{\partial \Omega} \varpi(x-\xi) \cdot \psi(\xi) \mathrm{da} \xi
\end{gathered}
$$

and is a solution to Stokes' Equations $(2)_{1,2}$ in $\mathbb{R}^{4} \backslash \partial \Omega$. The trace of $(7)_{1}$ on $\partial \Omega$ is a continuous operator

$$
\mathcal{S}: L^{q}(\partial \Omega) \rightarrow W^{1, q}(\partial \Omega)
$$

For $q=2, \mathcal{S}$ is Fredholm with index zero and $\operatorname{Kern} \mathcal{S}=\operatorname{Kern} \mathcal{S}^{\prime}=\{\boldsymbol{n}\},[2,3]$ where $^{3}$

$$
\mathcal{S}^{\prime}: W^{-1,2}(\partial \Omega) \rightarrow L^{2}(\partial \Omega)
$$


is the adjoint of $\mathcal{S}$. Hence, if $\boldsymbol{a} \in L^{2}(\partial \Omega)$, then there is $\boldsymbol{\psi} \in W^{-1,2}(\partial \Omega)$ such that the pair

$$
\begin{aligned}
& \boldsymbol{u}_{s}(x)=\boldsymbol{v}[\boldsymbol{\psi}]+\boldsymbol{\sigma}(x) \\
& p_{s}(x)=P[\boldsymbol{\psi}](x),
\end{aligned}
$$

is the unique solution to (2), which vanishes at infinity and takes the value $\boldsymbol{a}$ on $\partial \Omega$ according to $(3),[2,3]$ where

$$
\boldsymbol{\sigma}(x)=\frac{1}{\omega} \sum_{i=1}^{m} \frac{\left(x_{i}-x\right)}{\left|x-x_{i}\right|^{4}} \int_{\partial \Omega_{i}} \boldsymbol{a} \cdot \boldsymbol{n} .
$$

By classical stability results, the Fredholm property of (8) can be extended in a neighborhood of $(2-\epsilon, 2+\epsilon)$, with $\epsilon$ depending on $\partial \Omega$. If $\partial \Omega$ is of class $C^{1}$, then $(8)$ is Fredholm for all $q \in(1,+\infty)$. By results of [4], if $\boldsymbol{a} \in L^{3}(\partial \Omega)$, then the above solution belongs to $L^{4}(\Omega)$ and

$$
\left\|\boldsymbol{u}_{s}\right\|_{L^{4}(\Omega)} \leq \gamma\|\boldsymbol{a}\|_{L^{3}(\partial \Omega)}
$$

\section{Proof of Theorem 1}

Following [5], let $\boldsymbol{a}_{\epsilon} \in W^{1,2}(\partial \Omega)$ be such that

$$
\int_{\partial \Omega}\left|\boldsymbol{a}-\boldsymbol{a}_{\epsilon}\right|^{3}<\epsilon
$$

for small positive $\epsilon$. Let $\boldsymbol{u}_{\boldsymbol{s} \epsilon}$ be the solution to the Stokes problem with boundary value $\boldsymbol{a}-\boldsymbol{a}_{\epsilon}$. For $\boldsymbol{u} \in L^{4}(\Omega)$, denote by $\mathcal{K}[\boldsymbol{u}]$ the solution to the Stokes problem with boundary value $-\operatorname{tr}_{\mid \partial \Omega} \mathcal{V}[\boldsymbol{u}]$, where

$$
\mathcal{V}[\boldsymbol{u}](x)=\int_{\Omega} \mathfrak{u}(x-y)(\boldsymbol{u} \cdot \nabla \boldsymbol{u})(y) \mathrm{dv}_{y} .
$$

Consider the functional equation

$$
\boldsymbol{u}^{\prime}(x)=\boldsymbol{u}_{s \epsilon}+(\mathcal{K}+\mathcal{V})[\boldsymbol{u}]
$$

By virtue of (11), (12) we see that $\boldsymbol{u}^{\prime}$ is a contraction in $L^{4}(\Omega)$. Hence, it follows that (13) has a fixed point $\boldsymbol{u}_{\epsilon}$ which is a solution to (1) 1,2 taking the value $\boldsymbol{a}-\boldsymbol{a}_{\epsilon}$ on $\partial \Omega$ and such that

$$
\left\|\boldsymbol{u}_{\epsilon}\right\|_{L^{4}(\Omega)} \leq c \epsilon
$$

Let $\boldsymbol{v}_{s}$ be the solution to the Stokes problem with boundary value $\boldsymbol{a}_{\epsilon}$ :

$$
\boldsymbol{v}_{\epsilon}=\boldsymbol{v}[\psi]+\boldsymbol{\sigma}_{\epsilon}, \quad \boldsymbol{\sigma}_{\epsilon}(x)=-\frac{\boldsymbol{x}}{\omega|\boldsymbol{x}|^{4}} \int_{\partial \Omega} \boldsymbol{a}_{\epsilon} \cdot \boldsymbol{n} .
$$

Let us look for a solution $w \in W_{0}^{1,2}\left(\Omega_{R}\right)$ to

$$
\begin{aligned}
v \Delta \boldsymbol{w}-\left(\boldsymbol{u}_{\epsilon}+\boldsymbol{v}_{s}+\boldsymbol{w}\right) \cdot \nabla\left(\boldsymbol{v}_{s}+\boldsymbol{w}\right)-\left(\boldsymbol{w}+\boldsymbol{v}_{s}\right) \cdot \nabla \boldsymbol{u}_{\epsilon}-\nabla Q=\mathbf{0} \\
\operatorname{div} \boldsymbol{w}=0,
\end{aligned}
$$

in $\Omega_{R}=\Omega \cap S_{R}$, for large $R$. To this end, it is sufficient to show that the set of all solutions to (16) are bounded in $W^{1,2}(\Omega)$ (see, e.g. [1]). Following a classical reductio ad absurdum argument of J. Leray [6], let us suppose that a sequence of solutions $w_{k} \in W_{0}^{1,2}(\Omega)$ to (16) exists such that

$$
\lim _{k \rightarrow+\infty} J_{k}=+\infty, \quad J_{k}=\left\|\nabla \boldsymbol{w}_{k}\right\|_{L^{2}(\Omega)}
$$


Setting $\boldsymbol{w}_{k}^{\prime}=\boldsymbol{w}_{k} / J_{k}$, a straightforward argument shows that $\boldsymbol{w}_{k}^{\prime}$ tends strongly in $L^{q}\left(\Omega_{R}\right), q<4$, and weakly in $W^{1,2}\left(\Omega_{R}\right)$, to a field $\boldsymbol{w}^{\prime} \in W_{0}^{1,2}(\Omega)$, with $\left\|\nabla \boldsymbol{w}^{\prime}\right\|_{\left.L^{2} \Omega\right)} \leq 1$, which satisfies the Euler equations

$$
\begin{aligned}
\boldsymbol{w}^{\prime} \cdot \nabla \boldsymbol{w}^{\prime}+\nabla Q^{\prime}=\mathbf{0} & \text { in } \Omega_{R} \\
\operatorname{div} \boldsymbol{w}^{\prime}=0 & \text { in } \Omega_{R},
\end{aligned}
$$

for some field $Q^{\prime} \in W^{1,4 / 3}\left(\Omega_{R}\right)$ constant on $\partial \Omega$ and $\partial S_{R}$ (say, $Q_{0}$ on $\partial \Omega$ and $Q_{R}$ on $\partial S_{R}$ ) and

$$
v=\int_{\Omega} \boldsymbol{w}^{\prime} \cdot \nabla \boldsymbol{w}^{\prime} \cdot\left(\boldsymbol{u}_{\epsilon}+\boldsymbol{v}_{s}\right) .
$$

Let us extend $\boldsymbol{w}^{\prime}$ to $\Omega$ by setting $\boldsymbol{w}^{\prime}=\mathbf{0}$ in $C S_{R}$. Since

$$
\begin{aligned}
\left|\int_{\Omega} \boldsymbol{w}^{\prime} \cdot \nabla \boldsymbol{w}^{\prime} \cdot\left(\boldsymbol{u}_{\epsilon}+\boldsymbol{v}_{s}\right)\right| & \leq\left\|\boldsymbol{u}_{\epsilon}\right\|_{L^{4}(\Omega)}\left\|\boldsymbol{w}^{\prime}\right\|_{L^{4}(\Omega)}\left\|\nabla \boldsymbol{w}^{\prime}\right\|_{L^{2}(\Omega)} \leq c \epsilon, \\
\int_{\Omega} \boldsymbol{w}^{\prime} \cdot \nabla \boldsymbol{w}^{\prime} \cdot \boldsymbol{v}[\boldsymbol{\psi}] & =-\int_{\partial \Omega_{R}} \nabla Q^{\prime} \cdot \boldsymbol{v}[\boldsymbol{\psi}] \\
& =-Q_{R} \int_{\partial S_{R}} \boldsymbol{v}[\boldsymbol{\psi}] \cdot \boldsymbol{n}-Q_{0} \int_{\partial \Omega} \boldsymbol{v}[\boldsymbol{\psi}] \cdot \boldsymbol{n}=0,
\end{aligned}
$$

and, taking into account that $\nabla \boldsymbol{w}^{\prime} \cdot \nabla \boldsymbol{w}^{\prime \top}\left|=\hat{\nabla} \boldsymbol{w}^{\prime}\right|^{2}-\left.\tilde{\nabla} \boldsymbol{w}^{\prime}\right|^{2}$, with $\hat{\nabla} \boldsymbol{w}^{\prime}, \tilde{\nabla} \boldsymbol{w}^{\prime}$ symmetric and skew parts of $\nabla \boldsymbol{w}^{\prime}$ respectively and $\left\|\nabla \boldsymbol{w}^{\prime}\right\|_{L^{2}(\Omega)}^{2}=2\left\|\tilde{\nabla} \boldsymbol{w}^{\prime}\right\|_{L^{2}(\Omega)}^{2}=2\left\|\hat{\nabla} \boldsymbol{w}^{\prime}\right\|_{L^{2}(\Omega)}^{2}$,

$$
\begin{aligned}
\left|\int_{\Omega} \boldsymbol{w}^{\prime} \cdot \nabla \boldsymbol{w}^{\prime} \cdot \boldsymbol{\sigma}_{\epsilon}\right| & =\left|\int_{\partial \Omega} \boldsymbol{a}_{\epsilon} \cdot \boldsymbol{n}\right|\left|\int_{\Omega} \frac{\nabla \boldsymbol{w}^{\prime} \cdot \nabla \boldsymbol{w}^{\prime \top}}{2 \omega|\boldsymbol{x}|^{2}}\right| \\
\leq & \left|\int_{\partial \Omega}\left(\boldsymbol{a}_{\epsilon}-\boldsymbol{a}\right) \cdot \boldsymbol{n}\right|\left|\int_{\Omega} \frac{\nabla \boldsymbol{w}^{\prime} \cdot \nabla \boldsymbol{w}^{\prime \top}}{2 \omega|\boldsymbol{x}|^{2}}\right| \\
& +\left|\int_{\partial \Omega} \boldsymbol{a} \cdot \boldsymbol{n}\right|\left|\int_{\Omega} \frac{\left|\hat{\nabla} \boldsymbol{w}^{\prime}\right|^{2}-\left|\tilde{\nabla} \boldsymbol{w}^{\prime}\right|^{2}}{2 \omega|\boldsymbol{x}|^{2}}\right| \leq c \epsilon+\mathcal{F}
\end{aligned}
$$

from (19) it follows

$$
v-c \epsilon-\mathcal{F} \leq 0
$$

Therefore, since $\epsilon$ can be chosen small as we want, we see that the hypothesis ad absurdum (17) implies that (4) is not true and this gives the desired uniform estimate. Set $R=k \in \mathbb{N}$ for $k \geq k_{0}$ and denote by $\left(\boldsymbol{w}_{k}, Q_{k}\right)$ a solution to (16). By repeating ad litteram, the above by contradiction argument (with obvious modification), we see that the sequence $\left\{\boldsymbol{w}_{k}\right\}$ is uniformly bounded in $D^{1,2}(\Omega)^{4}$ and this is sufficient to conclude that (16) has a solution $\boldsymbol{w} \in D_{0}^{1,2}(\Omega)$ in $\Omega$.[1,7] Clearly, the field $\boldsymbol{u}=\boldsymbol{u}_{\epsilon}+\boldsymbol{v}_{s}+\boldsymbol{w}$ gives the desired solution to (1). If $(\boldsymbol{u}+\boldsymbol{w}, p+Q)$ is another solution to (1), with $\boldsymbol{u}+\boldsymbol{w} \in L^{4}(\Omega)$ and $w \in D_{0}^{1,2}(\Omega)$, then a simple computation and Schwarz' inequality and Sobolev's inequality yield

$$
\begin{aligned}
v \int_{\Omega}|\nabla \boldsymbol{w}|^{2} & =\int_{\Omega} \boldsymbol{w} \cdot \nabla \boldsymbol{w} \cdot \boldsymbol{u} \leq\|\boldsymbol{u}\|_{L^{4}(\Omega)}\|\boldsymbol{w}\|_{L^{4}(\Omega)}\|\nabla \boldsymbol{w}\|_{L^{2}(\Omega)} \\
& \leq \frac{3}{4}\|\boldsymbol{u}\|_{L^{4}(\Omega)}\|\nabla \boldsymbol{w}\|_{L^{2}(\Omega)}^{2} .
\end{aligned}
$$

Hence if $3\|\boldsymbol{u}\|_{L^{4}(\Omega)}<4 v$, then $\boldsymbol{w}=\mathbf{0}$. Write $\boldsymbol{u}=\boldsymbol{u}_{s}+\boldsymbol{w}$, where $\boldsymbol{u}_{s}$ is the solution (10) to the Stokes problem with boundary datum $\boldsymbol{a}$, and assume $3\left\|\boldsymbol{u}_{s}\right\|_{L^{4}(\Omega)}<4 v$. Therefore, $\boldsymbol{w} \in D_{0}^{1,2}(\Omega)$ is a solution 
to the equations

$$
\begin{aligned}
v \Delta \boldsymbol{w}-\left(\boldsymbol{u}_{s}+\boldsymbol{w}\right) \cdot \nabla\left(\boldsymbol{u}_{s}+\boldsymbol{w}\right)-\nabla p=\mathbf{0} & \text { in } \Omega \\
\operatorname{div} \boldsymbol{w}=0 & \text { in } \Omega, \\
\boldsymbol{w}=\mathbf{0} & \text { on } \partial \Omega, \\
\lim _{x \rightarrow \infty} \boldsymbol{w}(x)=\mathbf{0} . &
\end{aligned}
$$

By a standard argument

$$
\begin{aligned}
v \int_{\Omega}|\nabla \boldsymbol{w}|^{2} & =\int_{\Omega} \boldsymbol{w} \cdot \nabla \boldsymbol{w} \cdot \boldsymbol{u}_{s}+\int_{\Omega} \boldsymbol{u}_{s} \cdot \nabla \boldsymbol{w} \cdot \boldsymbol{u}_{s} \\
& \leq \frac{3}{4}\|\boldsymbol{u}\|_{L^{4}(\Omega)}\|\nabla \boldsymbol{w}\|_{L^{2}(\Omega)}^{2}+\left\|\boldsymbol{u}_{s}\right\|_{L^{4}(\Omega)}^{2}\|\nabla \boldsymbol{w}\|_{L^{2}(\Omega)} .
\end{aligned}
$$

Hence

$$
\frac{4}{3}\left(v-\frac{3}{4}\left\|\boldsymbol{u}_{s}\right\|_{L^{4}(\Omega)}\right)\|\boldsymbol{w}\|_{L^{4}(\Omega)} \leq\left(v-\frac{3}{4}\left\|\boldsymbol{u}_{s}\right\|_{L^{4}(\Omega)}\right)\|\nabla \boldsymbol{w}\|_{L^{2}(\Omega)} \leq\left\|\boldsymbol{u}_{s}\right\|_{L^{4}(\Omega)}^{2} .
$$

By (22) and Minkowski inequality

$$
\|\boldsymbol{u}\|_{L^{4}(\Omega)} \leq\left\|\boldsymbol{u}_{s}\right\|_{L^{4}(\Omega)}+\|\boldsymbol{w}\|_{L^{4}(\Omega)} \leq\left\|\boldsymbol{u}_{s}\right\|_{L^{4}(\Omega)}+\frac{\left\|\boldsymbol{u}_{s}\right\|_{L^{4}(\Omega)}^{2}}{\frac{4}{3}\left(v-\frac{3}{4}\left\|\boldsymbol{u}_{s}\right\|_{L^{4}(\Omega)}\right)} .
$$

Hence it follows that (5) yields uniqueness in $L^{4}(\Omega)$. Note that by (11), (5) is implied by $\gamma\|\boldsymbol{a}\|_{L^{3}(\partial \Omega)}<$ $4 v / 3$ and

$$
\gamma\|\boldsymbol{a}\|_{L^{3}(\partial \Omega)}+\frac{\gamma^{2}\|\boldsymbol{a}\|_{L^{3}(\Omega)}^{2}}{\frac{4}{3}\left(v-\frac{3 \gamma}{4}\|\boldsymbol{a}\|_{L^{3}(\partial \Omega)}\right)}<\frac{4 v}{3} .
$$

Remark 1: It is clear that the proof of existence of a solution to $(1)$ in $L^{4}(\Omega)$ requires only that the corresponding Stokes problem has a solution $\boldsymbol{u}_{s} \in L^{4}(\Omega)$. Hence, it follows that if $\partial \Omega$ is of class $C^{1,1}$, then Theorem 1 can be extended to boundary data $\boldsymbol{a} \in W^{-1 / 4,4}(\partial \Omega)$.[3]

\section{Some remarks in higher dimensions}

If $\Omega$ is an exterior domain of $\mathbb{R}^{m}(m>3)$ of class $C^{1}$, then for $\boldsymbol{a} \in L^{m-1}(\partial \Omega)$ the Stokes problem

$$
\begin{aligned}
& v \Delta \boldsymbol{u}-\nabla p=\mathbf{0} \text { in } \Omega \\
& \operatorname{div} \boldsymbol{u}=0 \quad \text { in } \Omega \text {, } \\
& \boldsymbol{u}=\boldsymbol{a} \text { on } \partial \Omega \text {, } \\
& \lim _{x \rightarrow \infty} \boldsymbol{u}(x)=\mathbf{0},
\end{aligned}
$$

has a solution $\boldsymbol{u}_{s} \in L^{m}(\Omega)$ and $\left\|\boldsymbol{u}_{s}\right\|_{L^{m}(\Omega)} \leq \gamma\|\boldsymbol{a}\|_{L^{m-1}(\partial \Omega)}$ (see [3]). For $\boldsymbol{u} \in L^{m}(\Omega)$ consider the functional equation

$$
\boldsymbol{u}^{\prime}(x)=\boldsymbol{u}_{s}+(\mathcal{K}+\mathcal{V})[\boldsymbol{u}]
$$

in $L^{m}(\Omega)$, where the operator $\mathcal{K}+\mathcal{V}$ is defined in the proof of Theorem 1 . Taking into account that

$$
\|(\mathcal{K}+\mathcal{V})[\boldsymbol{u}]\|_{L^{m}(\Omega)} \leq c\|\boldsymbol{u}\|_{L^{m}(\Omega)}^{2},
$$

If $\|\boldsymbol{a}\|_{L^{m-1}(\partial \Omega)}$ is sufficiently small, then $\boldsymbol{u}^{\prime}$ is a contraction in a ball of $L^{m}(\Omega)$ and the fixed point of (24) is a $C^{\infty}$ solution to (1). Moreover, if $\boldsymbol{u}+\boldsymbol{w} \in L^{m}(\Omega)$ is another solution to (1), then by Schwarz's inequality and Sobolev's inequality

$$
v \int_{\Omega}|\nabla \boldsymbol{w}|^{2}=\int_{\Omega} \boldsymbol{w} \cdot \nabla \boldsymbol{w} \cdot \boldsymbol{u} \leq \frac{(m-1)}{(m-2) \sqrt{m}}\|\boldsymbol{u}\|_{L^{m}(\Omega)}\|\nabla \boldsymbol{w}\|_{L^{m}(\Omega)}^{2} .
$$


Hence, it follows that the above solution is unique in the ball

$$
\|\boldsymbol{u}\|_{L^{m}(\Omega)}<\frac{\nu \sqrt{m}(m-2)}{m-1} .
$$

It is clear that for domains of class $C^{1}$ we can repeat the argument in the proof of Theorem 1 to see that for $\boldsymbol{a} \in L^{m-1}(\Omega)$ and fluxes obeying a condition of the type (4), then the Equations (1) have a solution $\boldsymbol{u}=\boldsymbol{u}_{\epsilon}+\boldsymbol{v}_{s}+\boldsymbol{w}$, with $\boldsymbol{u}_{\epsilon}$, $\boldsymbol{v}_{\mathcal{s}}$ regular in $\Omega$ and $\boldsymbol{w} \in D_{0}^{1,2}(\Omega)$. Up to date, we have not general results assuring that $w$ is regular.

\section{Notes}

1. For the main notation, we follow the monograph.[1]

2. See [3] and Section 2.

3. In such a case, (7) has to be understood as the value of the functional $\psi$ at $\mathcal{U}$.

4. $D^{1,2}(\Omega)=\left\{\varphi \in L_{\text {loc }}^{1}(\Omega):\|\nabla \varphi\|_{L^{2}(\Omega)}<+\infty\right\}$ and $D_{0}^{1,2}(\Omega)$ is the completion of $C_{0}^{\infty}(\Omega)$ with respect to $\|\nabla \varphi\|_{L^{2}(\Omega)}$.

\section{Disclosure statement}

No potential conflict of interest was reported by the author.

\section{References}

[1] Galdi GP. An introduction to the mathematical theory of the Navier-Stokes equations. Steady-state problems. New York: Springer; 2011.

[2] Mitrea M, Taylor M. Navier-Stokes equations on Lipschitz domains in Riemannian manifolds. Math. Ann. 2001;321:955-987.

[3] Russo R. On Stokes' problem. In: Rannacher R, Sequeira A, editors. Advances in mathematica fluid mechanics. Berlin: Springer-Verlag; 2010. p. 473-511.

[4] Shen Z. A note on the Dirichlet problem for the Stokes system in Lipschitz domains. Proc Amer Math Soc. 1995; 123:801-811.

[5] Marušić-Paloka E. Solvability of the Navier-Stokes system with $L^{2}$ boundary data. Appl Math Optim. 2000;41:365-375.

[6] Leray J. Étude de diverses équations intégrales non linéaire et de quelques problèmes que pose l'hydrodynamique [Study of different nonlinear integral equations and of some problems related to hydrodynamics]. J Math Pures Appl. 1933;12:1-82.

[7] Temam R. Navier-Stokes equations. Amsterdam: North-Holland; 1977. 